\title{
USE OF DATA DRIVEN MODELS IN PREDICTION: AN INTERDISCIPLINARY APPROACH FOR ANALYZING DATA.
}

\author{
Dhane Neeta $\mathrm{K}^{1}$
}

\begin{abstract}
In the last two decades, the rapid implementation and smart use of Information technology in all the domains of business sector, education, medical science, engineering, space and government offices has produce a vast amount of data. The increase in collection and systematic storage of data further promote the researchers and data scientist's to use the data for finding the hidden information in it by using data driven models. The use of data driven models in prediction has an interdisciplinary approach. The data scientist should posses the pure knowledge of statistics as well as computer science. Further the finding of the data analysis must help the society and hence he should have a good psychological thought for doing the data analysis. The paper aims to underline the importance of an interdis ciplinary approach for using the data driven models in prediction.
\end{abstract}

Keywords: Data driven models, data mining, interdisciplinary approach.

\section{INTRODUCTION}

The rapid and smart use of Information Technology in business has improved the ways to collect, store and analyze the data. The overall method of collecting and analyzing data has been drastically changed in the due course of time. The reasons behind it are many, out of which one is the use of database management system (DBMS) to store the huge amount of data and the various new edge software's to analyze the stored data. Earlier the data collected used to small in size, also it was localized data and because of such limitations the results incurred after analyzing the data doesn't affect the decision strategies. Now a days in the age of cut throat competition among the business rivals the data generated through the day to day transactions is a rich source of Knowledge. So we can frame it as "Knowledge in Data". The process of extraction of knowledge from the database is known as Data Mining. Data mining involves many different algorithms which examine the data and determine a model that is closest to the characteristics of the data being examined. Two types of models are used in data mining i.e Predictive model \& Descriptive model. A predictive model makes a prediction about the values of the data using known results found from different data. A descriptive model identifies patterns or relationships in data. Predictive modeling is often defined as a process that builds on data mining and probability theory for the purpose of forecasting outcomes. Every model contains a number of predictors, that is, variables that are likely to control or influence future outcomes. After collecting data on relevant variables, a statistical model can be formulated. Regression models were the first statistical models developed for the purpose of making prediction. The next class of prediction models is in the domain of time series. A time series is a sequence of observations on the variable of interest observed at time points that form a lattice set on the real line. There is only one variable and its values from the past are used for predicting its values in the future by fitting an appropriate statistical model.

\footnotetext{
${ }^{1}$ Research Scholar, JJTU Rajasthan.
} 


\section{DATA DRIVEN MODELS.}

Data driven modelling (DDM) is based on analysing the data about a system, in particular finding connections between the system state variables (input, internal and output variables) without explicit knowledge of the physical behavior of the system. These methods represent large advances on conventional empirical modelling and include contributions from the following overlapping fields:

Artificial intelligence (AI), which is the overarching study of how human intelligence can be incorporated into computers.

Computational intelligence (CI), which includes neural networks, fuzzy systems and evolutionary computing as well as other areas within AI and machine learning.

Soft computing (SC), which is close to CI, but with special emphasis on fuzzy rule-based systems induced from data.

Machine learning (ML), which was once a sub-area of AI that concentrates on the theoretical foundations used by CI and SC.

Data mining (DM) and knowledge discovery in databases (KDD) are focused often at very large databases and are associated with applications in banking, financial services and customer resources management. DM is seen as a part of a wider KDD. Methods used are mainly from statistics and ML.

Intelligent data analysis (IDA), which tends to focus on data analysis in medicine and research and incorporates methods from statistics and ML. Data-driven modelling is therefore focused on CI and ML methods that can be used to build models for complementing or replacing physically based models. A machine-learning algorithm is used to determine the relationship between a system's inputs and outputs using a training data set that is representative of all the behavior. Once the model is trained, it can be tested using an independent data set to deter-mine how well it can generalise to unseen data.

\section{INTERDISCIPLINARY APPROACH IN DATA MINING.}

The field of Data Mining has evolved from many several antecedents, such as Machine Learning, Statistics, and Decision support. Machine Learning has contributed many important algorithms for recognizing patterns in data. Statistics has provided a required background for data mining. Statisticians understand the best practices in modelling and experimental design. Over the past few decades, people have been gathering data into databases to make a better informed decisions. So we can say that Data Mining is a natural extension of such a vigorous effort.

Data Mining connects business needs to data. It is about understanding the customers and prospects, understanding products and markets, understanding suppliers and partners, understanding processes. Data mining is an interdisciplinary field bringing together techniques from machine learning, pattern recognition, statistics, databases, visualization, and neural networks.

\subsection{DATA MINING \& MACHINE LEARNING.}

The machine learning people come from the computer science and artificial intelligence worlds. The main focus of their efforts was on getting computers to display intelligence. Artificial Intelligence has opened a new avenue for making smart use of data in making smart use of data in making predictions. Instead of modeling data, the new methods attempt modeling relationships among variables and attributes. This modeling is based on data and not on any mathematical function that represents the population distribution of observed characteristics. This is the reason why traditional methods are often described as model based methods while the modern methods are called datadriven methods. Since these methods discover the possible relationships among data elements (that is, either variables or observations), they are also described as learning algorithms. If these learning algorithms are further automated, they belong to what is popularly known as the class of machine learning algorithms.

\subsection{DATA MINING \& STATISTICS.}

In early days, statistics meant information about states. Gradually, the focus continued to be on information but extended from states to anything and everything that can provide information. Nowadays, the term "Statistics" is used for both, sets of collected information as well as analytical work that involves statistical inference. Statisticians has always created methods to analyze data that has supported data mining. The statistical methods are usually developed in relation to the data being analyzed, but according to a conceptual reference paradigm. The characteristics that distinguishes data mining from statistical analysis is that statistical analysis traditionally concerns 
itself with analyzing primary data that has been collected to check specific research hypotheses, data mining can also concern itself with secondary data collected for other reasons. Furthermore, statistical data can be experimental data, but in data mining the data is typically observational data.

\section{CONCLUSIONS.}

The use of data driven models in prediction requires an interdisciplinary approach as it a collection of many different disciplines to find out the regular patterns in the collected data. Many new tools as well as software's are available in the market which make the job of data scientist easy. The new inventions and creation of automated software will provide faster and accurate results, but it is extremely true that persons with expertise in data mining would help the decision makers to choose the best one.

\section{REFERENCES.}

[1] Mastering Data Mining The Art and Science of Customer Relationship Management. Michael J.A.Berry,Gordan S Linoff. Wiley India.

[2] Data Mining Multimedia, Soft computing and Bio Informatics. Sushmita Mitra, Tinku Acharya. Wiley India.

[3] Applied Data Mining Statistical Methods for Business and Industry. Paolo Giudici. Wiley India.

[4] Data Mining Introductory and Advanced topics. Margaret H Dunham. Pearson.

[5] www.springer.com/cda/content/document/cda.../9783540343509-c1.pdf?SGWID...

[6] www.springer.com/cda/content/document/cda.../978354079880-c1.pdf?SGWID... 